\begin{tabular}{|c|l|}
\hline Title & Current timer switch in a GaA s based nanowire coupled with polyoxometal ate nanoparticle and conductive A FM tip \\
\hline Author(s) & Sasaki, Kentaro; Saito, Shunsuke; Kasai, Seiya \\
\hline Citation & $\begin{array}{l}\text { Japanese Journal of A pplied Physics (JJA P), 59(10), 105005 } \\
\text { https://doi.org/_0.35848/1347-4065/abb9c9 }\end{array}$ \\
\hline Issue Date & 2020-10-01 \\
\hline Doc URL & http://hdl.handle.net/2115/82829 \\
\hline Rights & ○ 2020 The Japan Society of A pplied Physics \\
\hline Type & article (author version) \\
\hline File Information & Sasaki_JJAP_20200719_revisedmanuscript.pdf \\
\hline
\end{tabular}

Instructions for use 


\title{
Current timer switch in GaAs-based nanowire coupled with polyoxometalate nanoparticle and conductive AFM tip
}

\author{
Kentaro Sasaki ${ }^{1,2}$, Shunsuke Saito ${ }^{1,2}$, and Seiya Kasai ${ }^{1,2,3^{*}}$ \\ 1 Research Center for Integrated Quantum Electronics, Hokkaido University, Sapporo 060- \\ 0813, Japan \\ 2 Graduate School of Information Science \& Technology, Hokkaido University, Sapporo 060- \\ 0814, Japan \\ 3 Center for Human Nature, Artificial Intelligence, and Neuroscience, Hokkaido University, \\ Sapporo 060-0810, Japan \\ *Corresponding: kasai@rciqe.hokudai.ac.jp
}

We demonstrate a current timer switch function in a GaAs-based nanowire electrostatically coupled with a polyoxometalate nanoparticle (POM NP) and a conductive atomic force microscopy (AFM) tip. The nanowire current associated with the charge state of the POM NP on the nanowire surface abruptly changed after several ten seconds from biasing the conductive tip. The current switch timing changed depending on the tip voltage. The timer switch function appeared when the tip approached the POM NP where the AFM phase image showed phase lag at high atmospheric humidity condition. We discuss the timer mechanism in terms of the configuration of the electro-mechanical potential of the cantilever and dynamic potential modulation by mobile ions and polarized molecules in the water-absorbed POM NP surface. 


\section{Introduction}

Molecular materials are expected to play important roles in future information devices and systems because of their small size and various functionalities ${ }^{1-7}$. Polyoxometalate (POM) is an attractive molecule for electronics because of its super-reduction capability ${ }^{8-13)}$; for example, a Keggin POM anion incorporating 12 molybdenum atoms can charge as many as 24 excess electrons ${ }^{14)}$. This distinctive electronic property was found to serve as a spiking neuron function in a very simple manner ${ }^{15)}$, which is quite difficult for conventional semiconductor devices; the charge dynamics in the solid-state semiconductor is immediately relaxed to the steady state due to the various energy dissipation processes ${ }^{16)}$ and the instability cannot be maintained in the device. Thus, in the current solid-state electronics, a circuit integrating several transistors and capacitors is necessary to generate spike train ${ }^{17-20)}$. The observed spiking in the POM NPcarbon nanotube (CNT) complex in ref. 15 suggests that the charge state of a POM molecule has unique and optimal dynamics. The spiking was hypothesized to be a result of the POM NP releasing all stored charges momentarily when the charges exceeded a threshold ${ }^{15}$. Experimental study using the simple network of the POM NP-CNT complex showed the generation of the stochastic current spikes ${ }^{21)}$. Recently, we investigated the charge dynamics of the individual POM NP using our dynamic charge detection system which includes a GaAsbased nanowire charge detector and a conductive atomic force microscopy (AFM) tip. The results suggested momentary transfer of a large amount of the charges in synchronized manner from the conductive tip to the POM NP ${ }^{22)}$. However, the detailed behavior and mechanism of the synchronized charge transfer of POMs in a NP have not been clarified yet. In this paper, we demonstrate a current timer switch in a GaAs-based nanowire coupled with a POM NP, which is based on the charge dynamics of POM. We observed the timer function using our dynamic charge detection system ${ }^{22-24)}$. In this study, atmospheric humidity of the system was precisely controlled to clarify the effect of the environment. We discuss the mechanism of the observed 
timer switch taking into account the role of the water molecules absorbed in the POM NP.

\section{Experimental procedure}

The schematics of the experimental setup are schematically shown in Fig. 1(a). The POM nanoparticles (NPs) were dispersed on the surface of the GaAs-based nanowire having a layer structure as shown in Fig. 1(b). The nanowire functioned as a high-sensitive and high-speed charge detector ${ }^{23-25)}$. The sample was set in an AFM system with a conductive-tip cantilever. Humidity in the enclosure was precisely controlled by the feedback control system as shown in Fig. 1(a), which was added to the previous setup ${ }^{22)}$. In this system, the sensitivity of the nanowire to the NP charge increases when the conductive tip approaches the NP and they are capacitively coupled. The equivalent circuit is shown in Fig. 1(c). The nanowire channel potential $\Delta V$ becomes sensitive to the charge $e$ in the NP when the conductive tip and the NP are capacitively coupled through an air-gap capacitance $C_{1}$ between the tip and the POM NP ${ }^{22-}$ 24),

$$
\Delta V=\frac{C_{1} e}{C_{1} C_{2}+C_{2} C_{3}+C_{3} C_{1}}
$$

where $C_{2}$ is the nanowire surface-channel capacitance and $C_{3}$ is the substrate capacitance. In our samples, $C_{3}$ was negligibly small compare to $C_{1}$ and $C_{2}$ because the thickness of the undoped GaAs substrate was $625 \mu \mathrm{m}$ and it was much larger the thicknesses of the dielectric parts in $C_{1}$ and $C_{2}$ with $10 \mathrm{~nm}$ order ${ }^{24)}$. Since a narrow metal AFM tip was used to form $C_{1}$, the charge could be detected in the local small portion. Then we could access the charge state in the individual NP.

In this study, we used a POM molecule with the composition of $\mathrm{Na}_{3}\left[\mathrm{PO}_{4} \cdot 12 \mathrm{MoO}_{3}\right] \cdot \mathrm{nH}_{2} \mathrm{O}$, as shown in Fig. 1(a), whose structure was derived from reported information ${ }^{26,27)}$. Activation energy for the electrical conduction of $\mathrm{Na}_{3}\left[\mathrm{PO}_{4} \cdot 12 \mathrm{MoO}_{3}\right] \cdot \mathrm{nH}_{2} \mathrm{O}$ was reported smaller than $\mathrm{H}_{3}\left[\mathrm{PO}_{4} \cdot 12 \mathrm{MoO}_{3}\right]$ and other compounds ${ }^{28)}$, thereby we expected the 
larger change of the electronic state in it by biasing the AFM tip than the others. POM molecules were dissolved in the $\mathrm{C}_{2} \mathrm{H}_{5} \mathrm{OH}$ solvent and they were dispersed on the nanowire surface using the drop casting technique. NPs were sparsely dispersed on the surface. The density of the POM NPs on the nanowire was controlled by adjusting the POM concentration in the solution ${ }^{22)}$. When the solvent was $\mathrm{C}_{2} \mathrm{H}_{5} \mathrm{OH}$, the nanowire current was largely unchanged before and after POM dispersion, whereas the current greatly decreased when the solvent was $\mathrm{H}_{2} \mathrm{O}^{22}$.

The GaAs-based nanowire was fabricated by electron beam lithography and wet chemical etching of the $\mathrm{AlGaAs} / \mathrm{GaAs}$ heterostructure. The details of the fabrication process can be seen in refs. 22 and 29. The measured electron mobility $\mu$ and sheet density $n_{\mathrm{s}}$ by the Hall measurement at room temperature were $7,200 \mathrm{~cm}^{2} \cdot \mathrm{V}^{-1} \cdot \mathrm{s}^{-1}$ and $7.8 \times 10^{11} \mathrm{~cm}^{-2}$, respectively. The high electron mobility contributes to the high charge sensitivity. Its low-noise property is more ideal than the MOS-type Si device ${ }^{29)}$ and CNT device ${ }^{30)}$. From our understanding of these devices, ${ }^{31-35)}$ we determined that the GaAs-based nanowire device possessed singleelectron charge sensitivity ${ }^{23,24)}$. The nanowire length $L$ was $4 \mu \mathrm{m}$. The width $W$ of the nanowires used in this study was between $300 \mathrm{~nm}$ and $480 \mathrm{~nm}$. The nanowire currents were systematically changed depending on $W$.

An optical-lever type AFM system (JPK instruments, NanoWizard) installed in an acoustic enclosure was used for contacting the POM NP and taking sample images. The cantilever was the standard conductive Si-based tip covered with PtIr. The tip was connected to the external DC voltage source. The sample was electrically connected to the external electric circuit through the specially designed probers equipped in the AFM system so the AFM and nanowire current measurement could be carried out at the same time. The nanowire current transient was measured and stored using a real-time spectrum analyzer with a low-noise current amplifier (LNA). The time resolution was $41 \mu \mathrm{s}$. In the current measurement, the vertical tip apex position was adjusted to $20 \mathrm{~nm}$ above the POM NP and the cantilever feedback control 
was turned off to avoid the LED light irradiation on the nanowire and POM NPs.

\section{Results}

Figure 2(a) shows an example of the AFM image taken of the prepared sample. The POM NPs were sparsely dispersed on the GaAs-based nanowire. The lateral size of the NPs was typically a few hundred nanometers in diameter and their height was approximately $15 \mathrm{~nm}$. Figures 2(b) and 2(c) show the energy dispersive X-ray spectroscopy (EDX) spectra from the POM crystal before dissolving and the POM NP dispersed on the nanowire, respectively. Considering the peaks from the GaAs nanowire in Fig. 2(c), the measured spectra indicated that the composition of POM was not largely changed after dispersion and $\mathrm{Na}$ was remained in the dispersed POM NP. We also checked the nanowire current before and after POM dispersion to verify the surface property of the nanowire was not changed by POM dispersion, because it is known that the surfaces of GaAs-based materials are chemically active and the surface electric property often becomes unstable. We confirmed that the current was almost unchanged before and after POM dispersion as reported previously ${ }^{22)}$ and the nanowire surface itself was not changed by the POM dispersion.

Figure 3(a) shows the nanowire currents for three different tip voltages measured at $38 \%$ atmospheric humidity. The horizontal axis of the plot represents the duration from the start of tip biasing. A clear current step was observed for each bias condition; the current was constant for about 60 seconds from the start of tip biasing and then decreased abruptly. This current timer switch was not seen in the nanowire with tetraphenylporphine (TPP) NPs ${ }^{23}$. On the other hand, similarities were observed between the current timer switch and the reported charge threshold property of the POM NP-CNT complex ${ }^{15}$. We considered that the observed current behavior was attributed to the multiple redox property of the POM molecules. We also verified that the current in the nanowire was unchanged before and after the experiment, 
because the negatively biased AFM tip induced the surface reaction on the nanowire in the high humidity condition and irreversible change of the surface property occurred, known as AFM tip-induced oxidation ${ }^{36)}$. We found that the current was returned to the initial value after the experiment, indicating that significant oxidation of the GaAs nanowire surface did not occur in the experiment even though negative bias was applied to the tip. The large current step height over $1 \mu \mathrm{A}$ at the high negative tip bias, as shown in Fig. 3(a), suggested the drastic change in the charge state of the POM NP. This distinct current change could not be explained by the electrostatic effect from the tip on the nanowire channel. Considering the charge sensitivity of the nanowire ${ }^{22-25)}$, over 200 electrons were necessary to charge the NP for explaining the observed current change around $1 \mu \mathrm{A}$.

We measured the current switch timing, corresponding to the set time in a timer, as a function of the tip voltage $V_{\text {Tip }}$ at humidity of $38 \%$ and $40 \%$. The results are shown in Fig. 3(b). Here, we examined two humidity condition to see whether humidity affected the timer function. We measured twice for checking the reproducibility at the several voltages where the two symbols appear at the same voltage in the plot. Although the absolute tip voltage ranges were different in the two experiments, the current switch timing increased in both cases when the tip bias was increased at constant humidity and the switching timing was over $30 \mathrm{~s}$. This result showed that the switch timing could be intentionally changed by changing the tip voltage. The shift of the voltage range in the two experiments may be due to the difference in the tip-NP capacitance, which was affected by the geometrical configuration and sizes of the NPs or the difference in the spring constant of the cantilever. On the other hand, it was unclear what mechanism determined the timing of the current switch. The switching timing of several ten seconds could not be explained by the time constant of the active redox of the POM NPs ${ }^{21,37)}$.

In our previous study, humidity was one of the indispensable parameters involved in the charge dynamics of the POM NP ${ }^{22}$. However, in this study, it was found that the observed 
function did not systematically depended on such macroscopic parameter, even though it was precisely controlled using the setup shown in Fig. 1(a). Then, we investigated the effect of humidity on the POM NP from the nanoscale point of view using the AFM phase image. Figure 4 shows AFM topographical and phase images taken of the POM-dispersed nanowire surfaces at atmospheric humidity of $30 \%, 40 \%$, and $60 \%$. Note that the sample at $30 \%$ humidity differed from the others. No notable correlation was observed between the topological images and humidity. On the other hand, the phase image showed bright contrast and a clear correlation with humidity. The bright parts corresponding to the phase lag appeared on the POM dispersed portions. The phase lag portions were sparsely distributed when humidity was low and increased as humidity increased. When humidity was $60 \%$, the small bright portions gathered and became large. The formation of a water meniscus is known to cause the phase lag in AFM 38). However, the bright parts were observed on the POM NP portions, but not on the GaAs nanowire surface. Then, it was considered that the POM NP surface absorbed water molecules from the atmosphere and its property was changed to cause the phase lag.

As shown in the bottom of Fig. 4, the total phase lag area at humidity of $40 \%$ was larger than that of $30 \%$. On the other hand, the phase lag portions were discretely and inhomogeneously distributed on the sample surface even when humidity was high. Then, we considered that the AFM phase lag portion directly correlated with the current time switch rather than macroscopic humidity value. To verify our hypothesis, we characterized the nanowire current in two opposite conditions; positioning the tip at a phase lag portion at low humidity of $30 \%$ and at a non-phase lag portion at relatively high humidity of $40 \%$ as shown in Figs. 5(a) and 5(b), respectively. The POM NP contacted with the tip was indicated by a dashed rectangular box in each figure. When the tip was set to the phase lag (bright) portion in the phase image, as shown in Fig. 5(a), the current timer switch could be observed with a relatively high probability. On the other hand, as shown in Fig. 5(b), when the tip was positioned on the 
NP with the in-phase (dark), the current timer switch was hardly seen even at high humidity. The obtained results verified that the timer function correlated with the phase lag of the POM $\mathrm{NP}$ and the water molecules involved in the function.

\section{Discussion}

A possible model for the observed timer function deduced from the obtained results is schematically shown in Figs. 6(a)-(c). This model was developed to explain the two characteristic features of the function: holding the initial current even under the negative tip bias and the sudden current drop several tens of seconds after the start of tip biasing. The tip voltage dependence and the effect of water molecules from the atmosphere are involved in the mechanism. The electrochemical property of POM molecules also plays a major role.

The constant nanowire current in the early stage of the time series, shown in Fig. 5, indicates that the electric field from the tip was screened by the POM NP and did not reach the nanowire channel. Then, the POM surface absorbing water molecules was metallic and terminated the lines of the electric force coming from the biased tip. The POM crystal absorbing water molecules and its electric conductivity were reported to rapidly increase when humidity increased from $20 \%$ to $60 \%{ }^{28)}$. The large and rapid current change is likely caused by the large charge injection from the electrical connection between the tip and the NP. In a previous study, we found that the electrostatic effect from the tip was not enough to cause the observed large current change ${ }^{22)}$ and a large charge injection into the POM NP was relevant for the present case.

To investigate the electrical connection between the tip and the POM NP, we focused on the electrostatic attractive force between the two; the electrostatic force mechanically moves the AFM cantilever downward and causes electrical breakdown, resulting in abrupt charge injection. In our experiment, the feedback control of the AFM cantilever's vertical position was 
canceled when the nanowire current was measured so the cantilever mechanically moved following the external force. Figure 6(d) shows the electromechanical potential in the vertical direction. The potential is calculated from the electrostatic force $F_{\mathrm{ES}}$ and restoring force $F_{\mathrm{R}}$ using the tip voltage and cantilever stiffness, respectively. $F_{\mathrm{R}}$ is given by $-k \cdot \Delta \mathrm{Z}^{2}$, where $k$ is the spring constant of the cantilever and $\Delta Z$ is the displacement of the tip. $F_{\mathrm{ES}}$ can be estimated by $F_{\mathrm{ES}}=C_{1} V_{\mathrm{Tip}}^{2} / 2(D-\Delta Z)$, where $C_{1}=2 \pi \varepsilon_{0} \varepsilon_{\mathrm{a}}\left[R^{-1}-(2(D-\Delta Z))^{-1}\right], \varepsilon_{\mathrm{a}}$ is the relative permittivity of air, $R$ is the effective radius of the tip apex, and $D$ is the initial distance between the tip and the POM NP. The parameter values used in Fig. 6(d) are $D=20 \mathrm{~nm}, R=15 \mathrm{~nm}, \varepsilon_{\mathrm{a}}=1$, and $k=1$. The result shows that the system has a potential barrier disappears when the vertical electric field is sufficiently increased. The large shift in the vertical position of the tip occurs when the potential barrier is removed by gradually increasing the electric field between the tip and the NP. The tip voltage dependence of the current switch timing due to the difference in the initial potential barrier height.

A possible explanation for the long-time evolution of the electric field between the tip and the POM NP is the delayed motion of mobile ions and/or polarized molecules in the POM NP surface. When POM $\left(\mathrm{Na}_{3}\left[\mathrm{PO}_{4} \cdot 12 \mathrm{MoO}_{3}\right]\right)$ absorbs water molecules, the $\mathrm{Na}^{+}$ion acts as a positive charge carrier ${ }^{28)}$. The movement of polarized water molecules in the POM crystal has a long time constant of more than $100 \mathrm{~s}^{39,40)}$ and may also be associated with the delay in the transient of the charge state in the NP surface. The positive mobile ions in the metallic POM NP surface terminate the lines of the electric force from the negatively biased tip (Fig. 6(a)). The ions then move to just below the tip due to the attractive force of the electric field. The increase in the positive charge concentration below the tip results in the increased density of the lines of the electric force (Fig. 6(b)). In accordance with Gauss's law, the strength of the electric field is represented by the density of the lines of the electric force, which are schematically shown by dotted lines in the figure, and the direction of the field is along the 
lines. Here, almost the lines of the electric force are directed along the tip-NP direction, then the vertical electric field is increased. Polarized water molecules also are redistributed to reach the equilibrium state, which can delay the convergence of the charge balance in the system. Consequently, the electric field between the tip and the POM NP continuously increases after applying a negative voltage to the tip. Meanwhile, the cantilever abruptly moves down when the potential barrier decreases and crosses the threshold. The electric conductivity between the tip and POM NP rapidly increases and a large number of electrons are momentarily injected into the NP (Fig. 6(c)), resulting in a large reduction of the nanowire current. The observed shift of the switching timing in Fig. 3(b) was contradict to this model. A possible explanation was the unexpected difference in the tip-NP capacitance $C_{1}$, which was affected by difference of the geometrical configuration, the sizes of the NPs, and/or the spring constant of the cantilever. In addition, the model indicates that the switch timing becomes short when humidity is increased, which is contradict to the result in Fig. 3(b). However, as shown in Fig. 5, high humidity does not always generate the phase lag of the POM NP. Further controlled experimentation is necessary to understand the observed behavior.

The model above consists of both electrochemical and electromechanical aspects, the mechanism of which is complicated. At present, the model includes several hypotheses and further research is needed to verify their validity. The most fundamental hypothesis is that the time scale of the timer switch function is dominated by the dynamics of the mobile ions and polarized molecules in the surface of the water-absorbing POM NP. This hypothesis would be verified by characterizing the current transient in the POM crystal surface and simultaneous measurements of the AFM tip current and the vertical tip position would support the model.

\section{Conclusions}

We showed a current timer switch in a GaAs-based nanowire with a polyoxometalate 
nanoparticle (POM NP) coupled with a metallic AFM tip. The nanowire current associated with the charge state of the POM NP spontaneously and abruptly decreased about 60 seconds after negative biasing the AFM tip, similar to a timer switch. The switch timing was changed in accordance with the tip voltage. This timer switch function was observed when the tip approached the POM NP with the phase lag in AFM phase image, which appeared at high atmospheric humidity condition. We discussed the timer mechanism in terms of the electromechanical potential in the cantilever and the dynamic potential modulated by mobile ions and polarized molecules in the conductive POM NP surface absorbing water molecules.

\section{Acknowledgments}

This work was partly supported by MEXT KAKENHI Grant Numbers JP25110001 and JP25110013, and JSPS KAKENHI Grant Number JP16K14240, Japan. 


\section{References}

1) A. Aviram and M. A. Ratner, Chemical Phys. Lett. 29, 277 (1974).

2) Molecular Architectonics, Ed. T. Ogawa (Springer, Switzerland, 2017).

3) Nanoelectronics and Information Technology, Ed. R. Waser (Wiley-VCH, Germany 2012) 3rd ed.

4) D. Xiang, X. Wang, C. Jia, T. Lee, and X. Guo, Chem. Rev. 116, 4318 (2016).

5) Z. J. Donhauser, B. A. Mantooth, K. F. Kelly, L. A. Bumm, J. D. Monnell, J. J. Stapleton, D. W. Price Jr., A. M. Rawlett, D. L. Allara1, J. M. Tour and P. S. Weiss, Science 292, 2303 (2001).

6) K. Moth-Poulsen and T. Bjørnholm, Nat. Nanotechnol. 4, 551 (2009).

7) T. A. Gschneidtner, Y. A. D. Fernadez, K. Moth-Poulsen, J. Mat. Chem. C 1, 7127 (2013).

8) M. T. Pope, A. Müller, Angew. Chem. Int. Ed. Engl. 30, 34 (1991).

9) A. E. Kuznetsov, Y. V. Geletii, C. L. Hill, K. Morokuma, and D. G. Musaev, J. Am. Chem. Soc. 131, 6844 (2009).

10) N. Kawasaki, H. Wang, R. Nakanishi, S. Hamanaka, R. Kitaura, H. Shinohara, T. Yokoyama, H. Yoshikawa, and K. Awaga, Angew. Chem. Int. Ed. 123, 3533 (2011).

11) J. P. Launay, J. Inorg. Nucl. Chem. 38, 807 (1976).

12) P. Gouzerh and M. Che, Actual. Chem. 298, 9 (2006).

13) J. J. Walsh, A. M. Bond, R. J. Forster, T. E. Keyes, Coord. Chem. Rev. 306, 217 (2016).

14) H. Wang, S. Hamanaka, Y. Nishimoto, S. Irle, T. Yokoyama, H. Yoshikawa, and K. Awaga, J. Am. Chem. Soc. 134, 4918 (2012).

15) H. Tanaka, M. Akai-Kasaya, A. TermehYousefi, L. Hong, L. Fu, H. Tamukoh, D. Tanaka, T. Asai, and T. Ogawa, Nat. Commun. 9, 2693 (2018).

16) L. Esaki and R. Tsu, IBM J. Res. Develop. 14, 61 (1970).

17) C. Mead and M. Ismail, Analog VLSI implementation of neural systems (Kluwer Academic Publishers, Boston, 1989). 
18) G. Indiveri, E. Chicca, adn R. Douglas, IEEE Trans Neural. Netw. 18, 253 (2007).

19) H. Tanaka, T. Morie, and K. Aihara, IEICE Trans. Fundamentals. E92-A, 1690 (2009).

20) G. Indiveri et al., Frontiers in Neuroscience 5, 73 (2011).

21) K. L. Goh, H. Fujii, A. Setiadi, Y. Kuwahara, and M. Akai-Kasaya, Jpn. J. Appl. Phys. 58, SIIB18 (2019).

22) K. Sasaki, S. Okamoto, S. Tashiro, T. Asai, and S. Kasai, Jpn. J. Appl. Phys. 58, SDDE13 (2019).

23) S. Okamoto, M. Sato, K. Sasaki, and S. Kasai, Jpn. J. Appl. Phys. 56, 06 GK02 (2017).

24) M. Sato, X. Yin, R. Kuroda, and S. Kasai, Jpn. J. Appl. Phys. 55, $02 B D 01$ (2016).

25) S. Inoue, R. Kuroda, X. Yin, M. Sato, and S. Kasai, Jpn. J. Appl. Phys. 54, 04DN07 (2015).

26) I.V. Kozhevnikov, in Polyoxometalate Molecular Science (Nano Science Series II), ed. J. J.

Borrás-Almenar, E. Coronado, A. Mueller, and M. T. Pope (Kluwer Academic Publishers, The Netherlands, 2003), p.351.

27) S. Ishimaru and N. Nakamura, Ber. Bunsengers, Phys. Chem. 97, 777 (1993).

28) N. Azuma, R. Ohtsuka, Y. Morioka, H. Kosugi, and J. Kobayashi, J. Mat. Chem. 1, 989 (1991).

29) T. Muramatsu, K. Miura, Y. Shiratori, Z. Yatabe, and S. Kasai, Jpn. J. Appl. Phys. 51, 06FE18 (2012)

30) Y. Lin, J. C. Tsang, M. Freitag, and P. Avouris, Nanotechnology 18, 295202 (2007).

31) S. Kasai, T. Hashizume, and H. Hasegawa, Jpn. J. Appl. Phys. 35, 6652 (1996).

32) M. Yumoto, S. Kasai, and H. Hasegawa, Appl. Surf. Sci. 190, 242 (2002).

33) M. Yumoto, S. Kasai, and H. Hasegawa, IOP Conf. Series 184, 213 (2005).

34) S. Kasai, T. Nakamura, and Y. Shiratori, Appl. Phys. Lett. 90, 203504 (2007).

35) Y. Shiratori and S. Kasai, Jpn. J. Appl. Phys. 47, 3086 (2008).

36) P. Avouris, R. Martel, T. Hertel, and R. Sandstrom, Appl. Phys. A 66, S659 (1998). 
37) A. Setiadi, H. Fujii, S. Kasai, K. Yamashita, T. Ogawa, T. Ikuta, Y. Kanai, K. Matsumoto, Y. Kuwahara, and M. Akai-Kasaya, Nanoscale 9, 10674 (2017).

38) S. Rozhok, P. Sun, R. Piner, M. Lieberman, and C. A. Mirkin, J. Phys. Chem. B 108, 7814 (2004).

39) A. V. Barana and Y. D. Lampeka, Theor. Exp. Chem. 43, 119 (2007).

40) T. Okuhara, S. Tatematsu, K. Y. Lee, and M. Misono, Bull. Chem. Soc. Jpn. 62, 717 (1989). 


\section{Figure captions}

Fig. 1 (a) Measurement setup and POM molecule structure, (b) layer structure of nanowire, and (c) equivalent circuit of the sample.

Fig. 2 (a) AFM topological image of POM dispersed GaAs-based nanowire and (b) EDX spectrum on POM dispersed GaAs nanowire.

Fig. 3 (a) Measured nanowire current transient at different tip voltages and (b) evaluated current holding time as a function of tip voltage in two different samples at humidity of $38 \%$ and $40 \%$.

Fig. 4 AFM topological and phase images taken on POM dispersed GaAs nanowire at humidity (a) $30 \%$, (b) $40 \%$, and (c) $60 \%$.

Fig. 5 Nanowire current transients and AFM phase images of the POM NP contacted with the AFM tip at humidity of (a) $30 \%$ and (b) $40 \%$. The POM NP was in the area indicated by a dashed rectangular box.

Fig. 6 Model for observed current timer switch: (a) after biasing tip, (b) on-going state, and (c) breakdown. In accordance with the Gauss's law, the strength of the electric field is represented by the density of the lines of the electric force indicated with dotted lines and the direction of the field is along the lines. (d) Calculated electro-mechanical potential of cantilever for different tip-NP effective electric field. 
(a)

POM: $\mathrm{Na}_{3}\left[\mathrm{PO}_{4} \cdot 12 \mathrm{MoO}_{3}\right] \cdot \mathrm{nH}_{2} \mathrm{O}$

Humidity-controlled enclosure

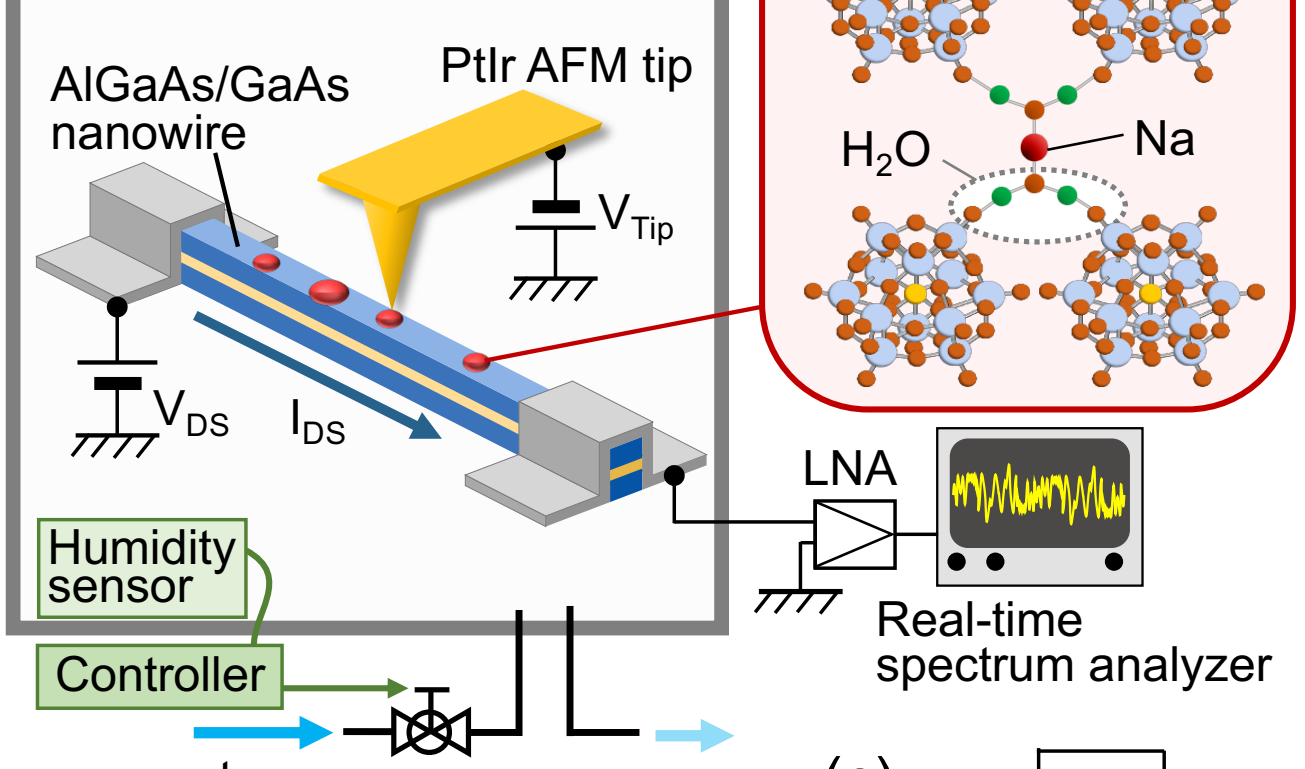
water vapor

(b)
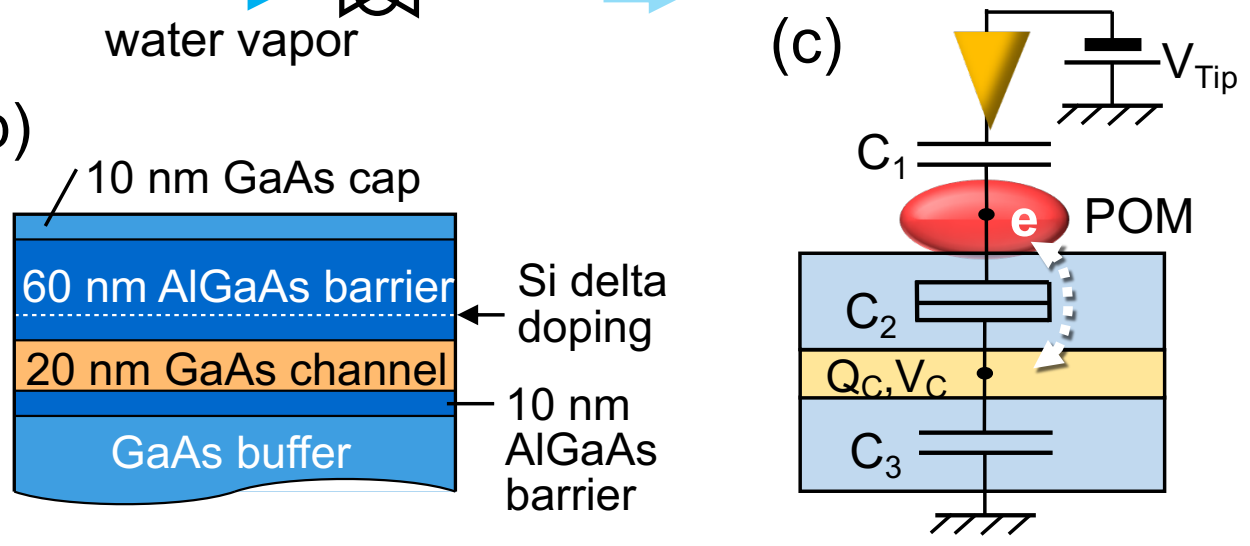

Figure 1 Kentaro Sasaki et al. 
(a)
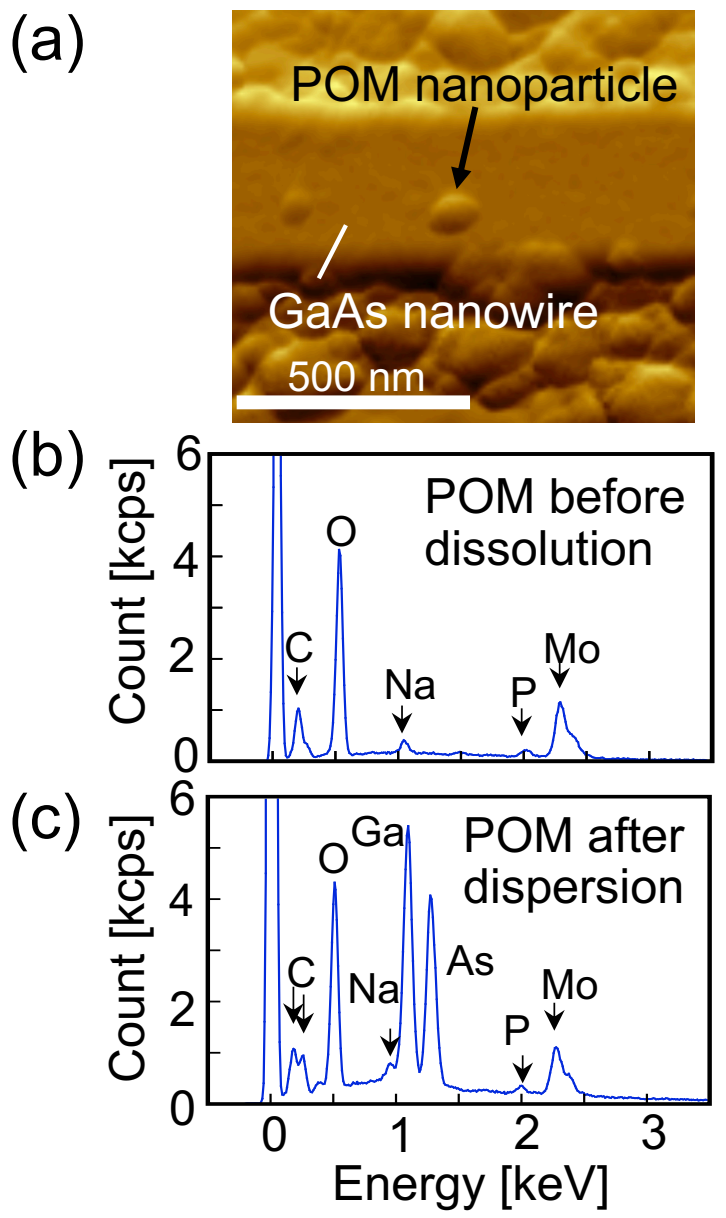

Figure 2 Kentaro Sasaki et al. 
(a)

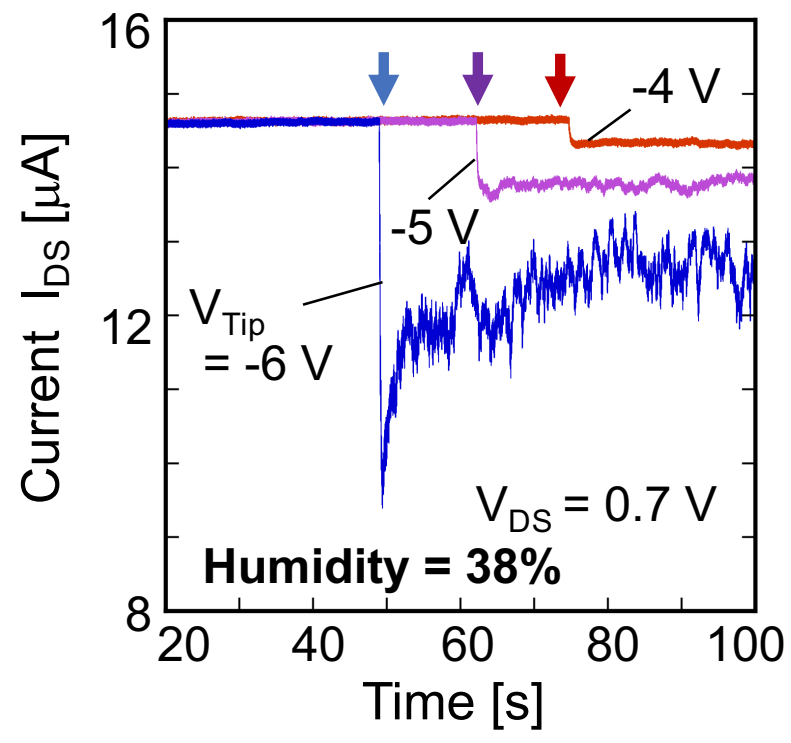

(b)

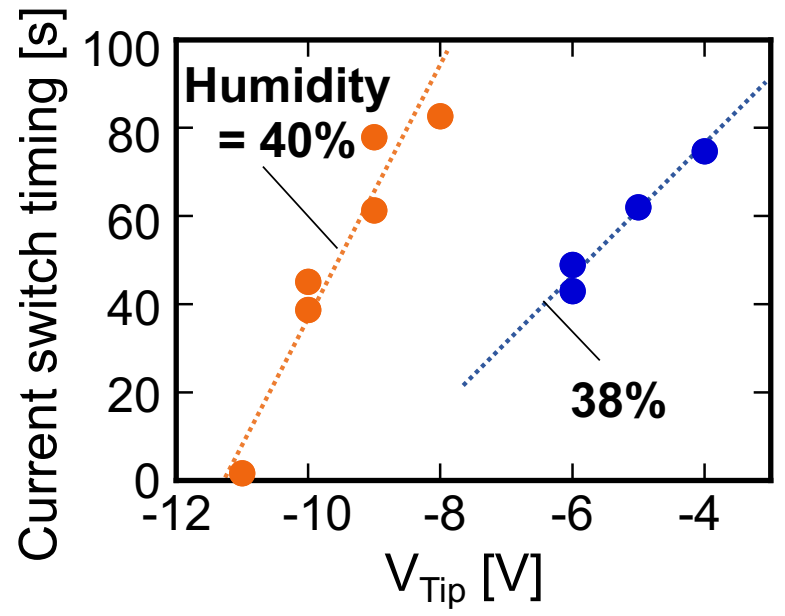

Figure 3 Kentaro Sasaki et al. 


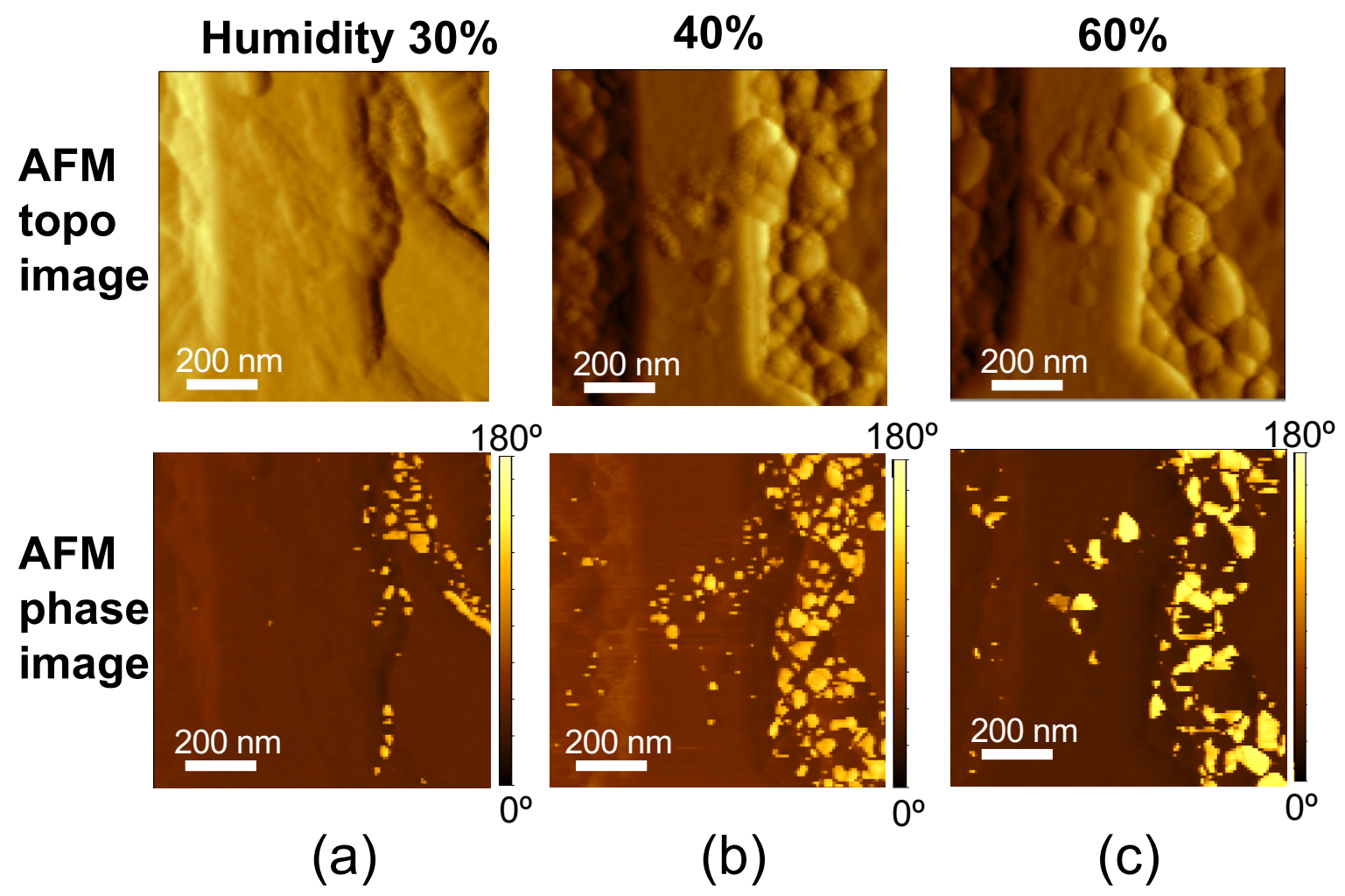

Figure 4 Kentaro Sasaki et al. 


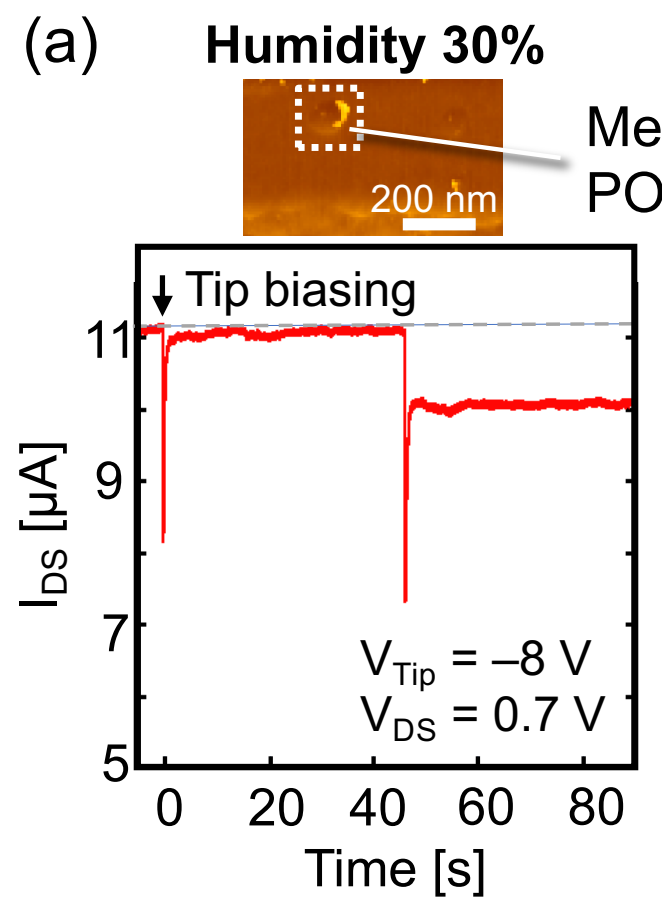

(b) Humidity $\mathbf{4 0} \%$ Measured NP

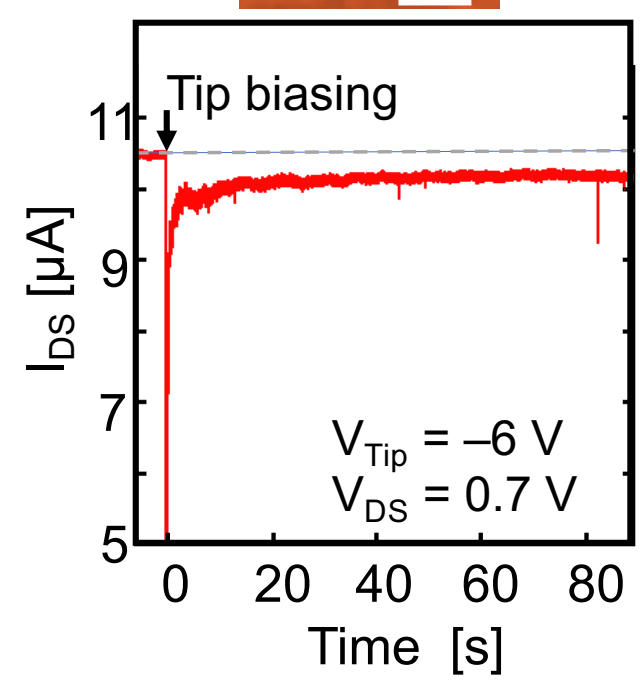

Figure 5 Kentaro Sasaki et al. 


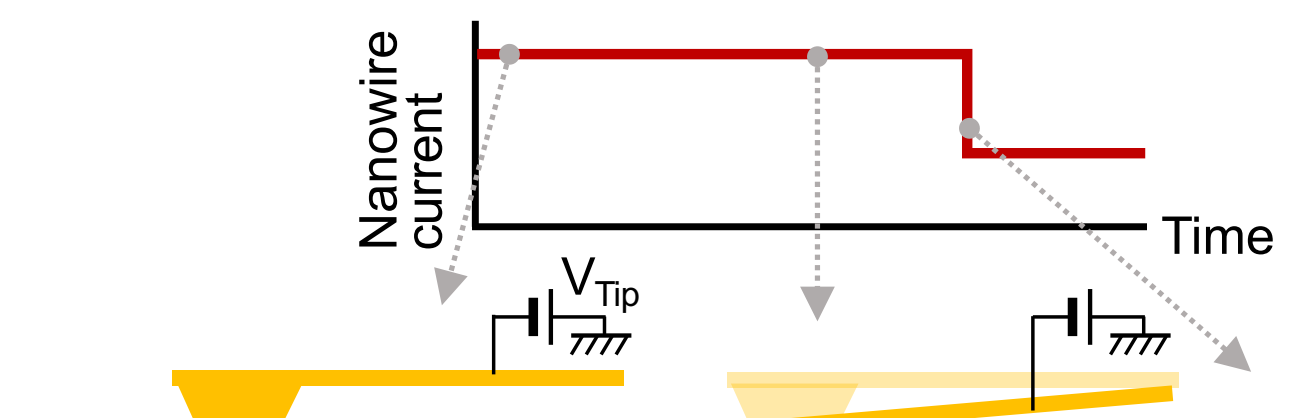

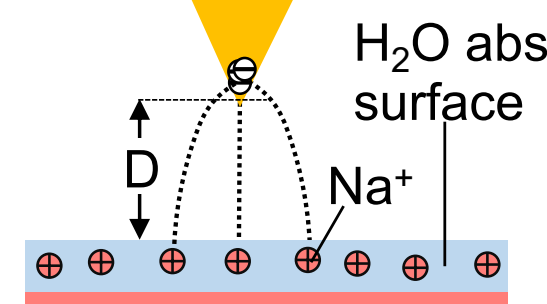

POM nanoparticle

(a)

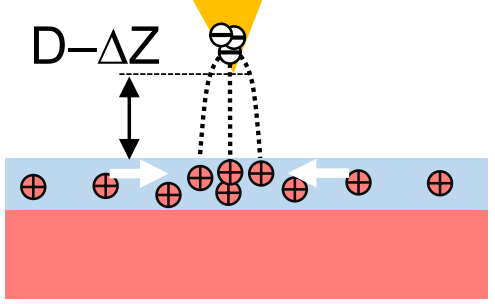

(b)

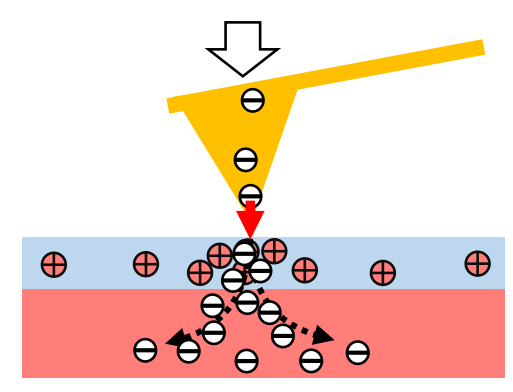

(c)

Energy [fJ]

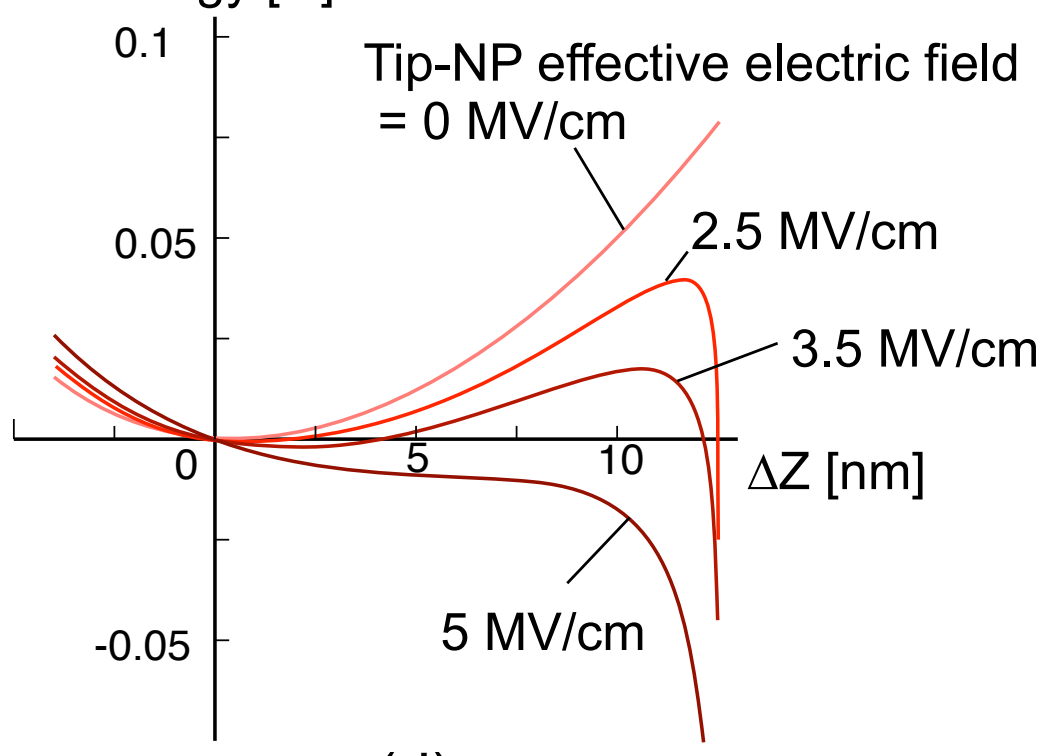

(d)

Figure 6 Kentaro Sasaki et al. 